\title{
Systematic review and meta-analysis of the efficacy and safety of novel monoclonal antibodies for treatment of relapsed/ refractory multiple myeloma
}

\author{
Tiantian Zhang ${ }^{1, *}$, Sen Wang ${ }^{1, *}$, Tengfei Lin ${ }^{2}$, Jingmei $\mathrm{Xie}^{1}$, Lina Zhao ${ }^{1}$, Zhuoru \\ Liang ${ }^{1}$, Yangqiu $\mathrm{Li}^{3,4}$ and Jie Jiang ${ }^{1,5}$ \\ ${ }^{1}$ College of Pharmacy, Jinan University, Guangzhou 510632, People's Republic of China \\ ${ }^{2}$ College of Food Science \& Nutritional Engineering, China Agricultural University, Beijing 100083, People's Republic of China \\ ${ }^{3}$ Department of Hematology, First Affiliated Hospital, Jinan University, Guangzhou 510632, People's Republic of China \\ ${ }^{4}$ Institute of Hematology, School of Medicine, Jinan University, Guangzhou 510632, People's Republic of China \\ ${ }^{5}$ Institute of Dongguan, Jinan University, Dongguan 523808, People's Republic of China \\ * These authors have contributed equally to this work \\ Correspondence to: Jie Jiang, email: jiangjie@jnu.edu.cn \\ Keywords: monoclonal antibody, elotuzumab, daratumumab, relapsed or refractory, multiple myeloma \\ Received: November 23, $2016 \quad$ Accepted: March 11, $2017 \quad$ Published: April 09, 2017 \\ Copyright: Zhang et al. This is an open-access article distributed under the terms of the Creative Commons Attribution License (CC-BY), \\ which permits unrestricted use, distribution, and reproduction in any medium, provided the original author and source are credited.
}

\section{ABSTRACT}

Although two newly launched monoclonal antibodies (mAbs), elotuzumab and daratumumab, performed well in patients with relapsed or relapsed/refractory multiple myeloma (RRMM), their efficacy and safety remain uncertain. We therefore performed a systematic review and meta-analysis of the most recent clinical trials that evaluated elotuzumab and/or daratumumab for the treatment of patients with RRMM. Our meta-analysis included 13 clinical trials with 2,402 patients participating. The overall response rate (ORR) was 57\% (95\% confidence interval [CI]: $38-76 \%$ ), and the at least very good partial response rate (VGPR) was 32\% (95\% CI: 1946\%). mAb-based regimens prolonged progression-free survival (PFS, hazard ratio: 0.52, 95\% CI: 0.36-0.75) compared to non-mAb-based regimens. Additionally, the efficacy of triplet regimens was superior to that of single or doublet regimens. The same trend was observed in a subgroup analysis of daratumumab and elotuzumab. The most common grade $3 / 4$ adverse events included neutropenia, lymphopenia, thrombocytopenia, anemia, leukopenia, pneumonia, and fatigue. Elotuzumab and daratumumab improved the ORR, at least VGPR, and PFS compared to non-mAb-based regimens. In a pooled analysis, both mAbs had promising efficacy and safety profiles, particularly in triplet regimens. The same trend was observed in daratumumaband elotuzumab-based regimens. Daratumumab triplet therapy (daratumumab, lenalidomide, and dexamethasone) was superior to other triplet regimens for the treatment of RRMM, and daratumumab monotherapy was more effective than either single agent in heavily pretreated MM patients, suggesting CD38 is an effective target for treatment of RRMM. Additional clinical studies of elotuzumab and daratumumab will be required to validate these results.

\section{INTRODUCTION}

Multiple myeloma is the second most common hematological malignancy. It is characterized by the proliferative disorder of plasma cells in the bone marrow with excessive monoclonal protein production [1]. There were approximately 30,330 new cases of multiple myeloma and 12,650 multiple myeloma-related deaths in the United States in 2016 [2]. Multiple myeloma accounts for approximately $1.8 \%$ of all cancers and $15 \%$ 
of all hematological malignancies in the United States [2]. Relapsed multiple myeloma is defined as previously treated multiple myeloma that has progressed and requires salvage therapy. Relapsed/refractory multiple myeloma (RRMM) is defined as disease that is nonresponsive to salvage therapy, or that progresses within 60 days of the last treatment in patients who have achieved a minimal response or better on prior therapy [3]. Standard treatment regimens for RRMM include proteasome inhibitors (PIs) such as bortezomib, and immunomodulatory drugs (IMiDs) such as lenalidomide alone or in combination with glucocorticoids (Table 1) [4]. Although these treatment regimens have improved patient survival, most patients eventually relapse following several lines of treatment [5]. Patients who are refractory to PIs and IMiDs, or who have received at least three prior lines of therapy (including a PI and an IMiD) are defined as heavily pretreated MM patients with highly refractory disease [6].

The Food and Drug Administration (FDA) approved pomalidomide (an IMiD) and carfilzomib (a PI) in 2013, which expanded the therapeutic options for multiple myeloma [7, 8]. Four additional drugs were approved in 2015: panobinostat, a histone deacetylase inhibitor [9], ixazomib (an oral PI) [10], elotuzumab (a monoclonal antibody $[\mathrm{mAb}]$ that targets the signaling lymphocytic activation molecule F7 [SLAMF7]) [11], and daratumumab (a mAb that targets CD38) [12]. These newly launched drugs are referred to as novel agents hereafter. The results of three recently published phase III randomized controlled trials (RCTs) have demonstrated that elotuzumab and daratumumab are promising therapeutics for multiple myeloma $[5,11,13]$. mAbs have been conjugated to various drugs, toxins, and radioisotopes, which improves efficacy. mAbs against interleukin-6, B-cell activating factor CD138, Dickkopf 1 (DKK1), receptor activator of nuclear factor- $\kappa \mathrm{B}$ ligand (RANKL), SLAMF7, and CD38 are currently in clinical development for the treatment of multiple myeloma [14]. Daratumumab is a human $\mathrm{IgG} 1 \mathrm{k}$ mAb that targets CD38, a cell surface protein that is highly expressed on myeloma cells and is involved in tumorigenesis, and promotes multiple myeloma cell cytotoxicity [15-17], phagocytosis $[17,18]$, induces apoptosis $[17,19]$, and depletes CD38positive immune regulatory $\mathrm{T}, \mathrm{B}$, and myeloid-derived suppressor cells [20]. Elotuzumab is a humanized IgG1 $\mathrm{mAb}$ that binds specifically to SLAMF7, also called CS1 (cell surface glycoprotein CD2 subset 1), which is highly expressed on the surface of multiple myeloma cells and on subsets of immune cells including natural killer (NK) cells, NK-like T cells, and a subset of CD8-positive T cells [21]. Elotuzumab promotes activation of NK cells and promotes cytotoxicity through the CD16 pathway [22]. Although both daratumumab and elotuzumab were approved by the FDA through the accelerated track, there is limited clinical data regarding the efficacy and toxicity of single or combination daratumumab- and elotuzumab-based regimens. However, several studies have provided evidence for the efficacy and safety of novel agents such as panobinostat [23], carfilzomib [24], and pomalidomide [24], as well as traditional agents including lenalidomide [25] and bortezomib [26], for RRMM treatment. Therefore, we performed a metaanalysis of RRMM patients treated with elotuzumab or daratumumab to evaluate the efficacy and safety of daratumumab and elotuzumab for RRMM treatment, and provide recommendations for the application of these agents in clinical practice. Additionally, we compared the efficacy and toxicity of daratumumab to other therapeutics in order to determine when it should be applied in clinical practice.

\section{RESULTS}

\section{Trial selection}

The process by which eligible trials were identified and selected for inclusion in our meta-analysis is illustrated in Figure 1. We identified 211 potentially relevant studies in our initial search of several databases. During the screening process, we excluded 161 articles, which included review articles, duplicate articles, retrospective studies, basic studies, and studies with no abstracts available. An additional 37 studies were excluded because they were trials that did not involve RRMM, elotuzumab or daratumumab, were retracted, or did not have sufficient trial data. The final meta-analysis consisted of 13 trials $(2,402$ patients $)$ that evaluated elotuzumab [11, 27-32] or daratumumab [5, 12, 13, 33-35] for the treatment of RRMM.

\section{Trial characteristics}

All 13 studies included in the meta-analysis were clinical trials that enrolled a total of 2,402 patients. The clinical trial characteristics are shown in Table 2.

\section{Efficacy}

\section{Efficacy of $\mathbf{m A b}$-based regimens}

All 13 studies (1,472 patients) included in our meta-analysis evaluated the efficacy of elotuzumab or daratumumab for RRMM according to the overall response rate (ORR) and the rate of achievement of at least very good partial response (VGPR). The ORR was 57\% (95\% confidence interval [CI]: $38-76 \%)$ and the at least VGPR rate was 32\% (95\% CI: 19-46\%) (Figure 2A, 2B). In a subgroup analysis (triplet/doublet/single regimen), the ORR was 76\% (95\% CI: 69-84\%) for 
Table 1: Traditional and novel agents for RRMM

\begin{tabular}{|l|l|l|c|}
\hline \multicolumn{1}{|c|}{ Agent } & \multicolumn{1}{|c|}{ Category } & \multicolumn{1}{c|}{ Target point } & Approval year by \\
\hline Traditional agents & & & 2005 \\
\hline Lenalidomide & IMiD & NA & 2003 \\
\hline Bortezomib & PI & 26S proteasome & NA \\
\hline Dexamethasone & Glucocorticoid & NA & 2015 \\
\hline Novel agents & & & 2015 \\
\hline Elotuzumab & mAb & SLAMF7 & 2015 \\
\hline Daratumumab & mAb & CD38 & 2015 \\
\hline Panobinostat & HDAC- inhibitor & HDAC & 2013 \\
\hline Ixazomib & PI & Peptide boronic acid proteasome & \\
\hline Carfilzomib & PI & Epoxyketone proteasome & 2013 \\
\hline Pomalidomide & IMiD & NA & \\
\hline
\end{tabular}

IMiD: immunomodulatory; PI: proteasome inhibitor; mAb: monoclonal antibody; HDAC- inhibitor: histone deacetylase inhibitor; SLAMF7: signaling lymphocytic activation molecule F7; NA: not available or no target points.

triplet regimens, suggesting they were more effective than doublet regimens (48\%, 95\% CI: $29-67 \%)$. The doublet regimens were more efficacious than single regimens (17\%, 95\% CI: 4-31\%). The same trend was observed in the at least VGPR. The at least VGPR was $48 \%(95 \%$ CI: $34-61 \%)$ for triplet regimens, $7 \%$ (95\% CI: $-2-17 \%)$ for doublet regimens, and 4\% (95\% CI: $0-8 \%$ ) for single regimens, indicating the triplet regimens were the most effective.

We next analyzed four head-to-head RCTs consisting of 1,865 RRMM patients that compared - to non-mAbbased triplet regimens (Figure 2C-E) [5, 11, 13, 32].

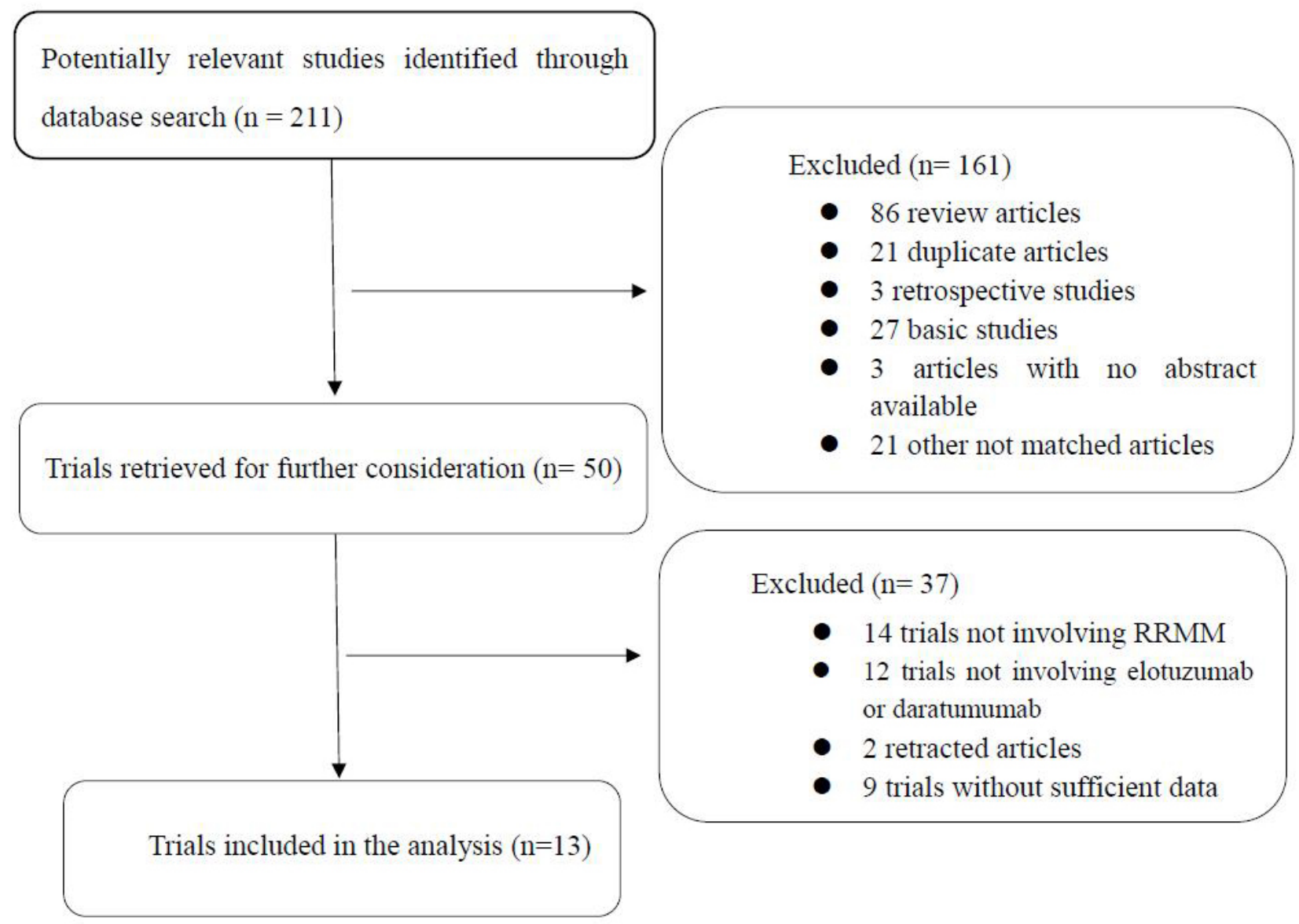

Figure 1: Identification and selection of the studies included in the meta-analysis. 
Table 2: Clinical trials information

\begin{tabular}{|c|c|c|c|c|c|c|c|c|c|c|}
\hline Study & Trial name & Phase & $\begin{array}{l}\text { Median age } \\
\text { (range) }\end{array}$ & \begin{tabular}{|l} 
Median \\
prior \\
therapy \\
(range) \\
\end{tabular} & $\begin{array}{l}\text { No. of } \\
\text { patients }\end{array}$ & Regimen & $\begin{array}{l}\text { Dose }(m g / \\
\text { kg) }\end{array}$ & $\begin{array}{l}\text { Follow-up } \\
\text { (months) }\end{array}$ & $\begin{array}{l}\text { Median } \\
\text { PFS time } \\
\text { (months) }\end{array}$ & $\begin{array}{l}\text { OS } \\
\text { rate } \\
\text {-year }\end{array}$ \\
\hline \multicolumn{11}{|l|}{ Elotuzumab } \\
\hline \multirow[t]{2}{*}{ Jakubowiak A(2016) [32] } & NCT01478048 & II & $65(25-82)$ & $1(1-3)$ & 77 & EVd & 10 & 15.9 & 9.7 & $73 \%-2$ \\
\hline & & & $65(25-85)$ & $1(1-3)$ & 75 & $\mathrm{Vd}$ & & 11.7 & 6.9 & $66 \%-2$ \\
\hline \multirow[t]{2}{*}{ Lonial S (2015) [11] } & ELOQUENT-2 & III & $67(37-88)$ & $2(1-4)$ & 321 & ERd & 10 & 24.5 & 19.4 & - \\
\hline & & & $66(38-91)$ & $2(1-4)$ & 325 & $\mathrm{Rd}$ & & 24.5 & 14.9 & - \\
\hline \multirow[t]{2}{*}{$\begin{array}{|ll|}\begin{array}{l}\text { RichardsonP } \\
{[27]}\end{array} & \mathrm{G}(2015) \\
\end{array}$} & 1703 & II & $60.6(39-77)$ & $2(1-3)$ & 36 & ERd & 10 & 21.2 & 32.49 & - \\
\hline & & & $63.3(41-82)$ & $2(1-3)$ & 37 & ERd & 20 & 16.8 & 25 & - \\
\hline Mateos(2016) [31] & NCT01632150 & II & $64(49-82)$ & $3(1-8)$ & 40 & ETd & 10 & - & 3.9 & $63 \%-1$ \\
\hline $\begin{array}{lll}\begin{array}{l}\text { Jakubowiak } \\
{[30]}\end{array} & \text { A } & \text { J(2012) } \\
\end{array}$ & NCT00726869 & I & $63(41-77)$ & $2(1-3)$ & 28 & $\mathrm{EV}$ & $2.5-20$ & - & 9.46(TTP) & - \\
\hline Lonial S(2012) [28] & NCT00742560 & $\mathrm{Ib}$ & $60(41-83)$ & $3(1-10)$ & 29 & ERd & $5,10,20$ & 16.4 & NR(TTP) & - \\
\hline Zonder J A(2012) [29] & NCT00425347 & $\mathrm{I}$ & $64.5(46-87)$ & $4.5(2-10)$ & 35 & $E$ & $0.5-20$ & - & - & - \\
\hline \multicolumn{11}{|l|}{ Daratumumab } \\
\hline \multirow[t]{2}{*}{ Palumbo A(2016) [5] } & CASTOR & III & $64(30-88)$ & $2(1-9)$ & 240 & DVd & 16 & 7.4 & NR & - \\
\hline & & & $64(33-85)$ & $2(1-10)$ & 234 & $\mathrm{Vd}$ & & 7.4 & 7.2 & - \\
\hline \multirow[t]{2}{*}{$\begin{array}{l}\text { Dimopoulos M A(2016) } \\
\text { [13] }\end{array}$} & POLLUX & III & $65(34-89)$ & $1(1-11)$ & 286 & DRd & 16 & 13.5 & NR & $92 \%-1$ \\
\hline & & & $65(42-87)$ & $1(1-8)$ & 283 & $\mathrm{Rd}$ & & 13.5 & 18.4 & $87 \%-1$ \\
\hline \multirow[t]{3}{*}{ Lokhorst H M(2015) [35] } & GEN501 & II & $59(38-76)$ & $4(3-10)$ & 30 & $\mathrm{D}$ & 8 & 16.9 & 2.4 & $77 \%-1$ \\
\hline & & II & $64(44-76)$ & $4(2-12)$ & 42 & $\mathrm{D}$ & 16 & 10.2 & 5.6 & $77 \%-1$ \\
\hline & & $\mathrm{I}$ & $61.5(42-76)$ & $6.3(2-12)$ & 32 & $\mathrm{D}$ & $0.005-24$ & - & - & - \\
\hline Lonial S(2016) [12] & SIRIUS & II & $63.5(31-84)$ & $5(2-14)$ & 106 & $\mathrm{D}$ & 16 & 9.3 & 3.7 & $65 \%-1$ \\
\hline \multirow[t]{2}{*}{ Plesner T(2016) [34] } & GEN503 & II & $59.5(41-76)$ & $2(1-3)$ & 32 & DRd & 16 & 15.6 & $\mathrm{NE}$ & $\begin{array}{r}90 \%- \\
1.5 \\
\end{array}$ \\
\hline & & $\mathrm{I}$ & $62(48-76)$ & $3(2-4)$ & 13 & DRd & $2-16$ & 23.5 & - & - \\
\hline Chari A(2015) [33] & NCT01998971 & $\mathrm{Ib}$ & $64(35-86)$ & $3.5(2-10)$ & 77 & $\mathrm{DPd}$ & 16 & 2.4 & - & - \\
\hline
\end{tabular}

E: elotuzumab; EVd: elotuzumab, bortezomib, and dexamethasone; Vd: bortezomib, dexamethasone; ERd: elotuzumab, lenalidomide, and dexamethasone; Rd: lenalidomide, dexamethasone; D: daratumumab; DRd: daratumumab, lenalidomide, and dexamethasone; DVd: daratumumab, bortezomib, and dexamethasone; DPd: daratumumab, pomalidomide, and dexamethasone; TTP: time to progression; NR: not reached.

Table 3 : Summary of response and survival outcomes from mAbs

\begin{tabular}{|c|c|c|c|c|c|c|c|c|}
\hline Regimen & No. of trials & $\begin{array}{l}\text { ORR } \\
(95 \% \text { CI })\end{array}$ & $P$ for ORR & $\begin{array}{l}\text { At least VGPR( }(95 \% \\
\text { CI) }\end{array}$ & $\begin{array}{l}P \text { for at } \\
\text { least VGPR }\end{array}$ & $\begin{array}{l}\text { OR of ORR } \\
(95 \% \text { CI) }\end{array}$ & $\begin{array}{l}\text { OR of at least } \\
\text { VGPR } \\
(95 \% \mathrm{CI})\end{array}$ & $\begin{array}{l}\text { HR of PFS } \\
(95 \% \mathrm{CI})\end{array}$ \\
\hline mAb & 13 & $57(38-76)$ & & $32(19-46)$ & & & & \\
\hline Triplet & 10 & $76(69-84)$ & $\mathrm{P}_{23}<0.000$ & $48(34-61)$ & $\mathrm{P}_{23}<0.000$ & $2.3(1.48-3.56)$ & $2.33(1.25-4.33)$ & $0.52(0.36-0.75)$ \\
\hline Doublet & 1 & $48(29-67)$ & $\mathrm{P}_{12}=0.002$ & $7(-2-17)$ & $\mathrm{P}_{12}=1$ & & & \\
\hline Single & 2 & $17(4-31)$ & $\mathrm{P}_{13}<0.000$ & $4(0-8)$ & $\mathrm{P}_{13}<0.000$ & & & \\
\hline Elotuzumab & 7 & $60(29-91)$ & & $29(15-44)$ & & & & \\
\hline Triplet & 5 & $73(61-84)$ & $\mathrm{P}_{23}<0.000$ & $38(27-48)$ & $\mathrm{P}_{23}=0.002$ & $1.63(1.03-2.58)$ & \begin{tabular}{|l|}
$1.33(0.97-1.77)$ \\
\end{tabular} & $0.70(0.59-0.84)$ \\
\hline Doublet & 1 & 48(29-67) & $\mathrm{P}_{12}<0.000$ & $7(-2-17)$ & $\mathrm{P}_{12}=0.192$ & & & \\
\hline Single & 1 & $1(-3-6)$ & $\mathrm{P}_{13}<0.000$ & $1(-3-6)$ & $\mathrm{P}_{13}<0.000$ & & & \\
\hline Daratumumab & 6 & $54(33-76)$ & & $35(13-57)$ & & & & \\
\hline Triplet & 4 & $81(71-91)$ & $\mathrm{Pd}<0.000$ & $59(44-75)$ & $\mathrm{Pd}<0.000$ & $3.25(2.31-4.56)$ & $3.75(2.88-4.88)$ & $0.38(0.30-0.48)$ \\
\hline Single $(16 \mathrm{mg} / \mathrm{kg})$ & 2 & $31(24-38)$ & & $11(6-16)$ & & & & \\
\hline
\end{tabular}

mAb: monoclonal antibody; OR: odds ratio; ORR: overall response ratio; VGPR: very good partial response; HR: hazard ratio; PFS: progression-free survival. $\mathrm{P}_{23}, \mathrm{P}_{12}, \mathrm{P}_{13}$ : The value between triplet and doublet, single and doublet, single and triplet, respectively. And when the $\mathrm{P}_{23}, \mathrm{P}_{12}$, or $\mathrm{P}_{23}$ is less than 0.0125 , the efficacy of ORR or at least VGPR between the group is significantly. Besides, when the Pd value is less than 0.05 , the efficacy of ORR or at least VGPR between the group is significantly. 
The overall weighted odds ratios [ORs] of the ORR and at least VGPR were 2.30 (95\% CI: 1.48-3.56) and 2.33 (95\% CI: 1.25-4.33), respectively, which suggested that $\mathrm{mAb}$-based regimens were more favorable. The overall progression-free survival (PFS) weighted hazard ratio [HR] was 0.52 (95\% CI: 0.36-0.75), which also indicated $\mathrm{mAb}$-based regimens were more effective than non-mAb regimens. Thus, although all mAb-based regimens (triplet/ doublet/single) displayed impressive efficacy profiles, $\mathrm{mAb}$-based triplet regimens were superior to doublet and single regimens based on the ORR and at least VGPR. In addition, $\mathrm{mAb}$-based triplet regimens were superior to non-mAb-based regimens based on the ORR, at least VGPR, and PFS.

\section{Efficacy of elotuzumab-based regimens}

There were seven studies (603 patients) that evaluated the efficacy of elotuzumab for the treatment of RRMM based on ORR and at least VGPR [11, 27-32]. The ORR was 60\% (95\% CI: 29-91\%) and the at least VGPR
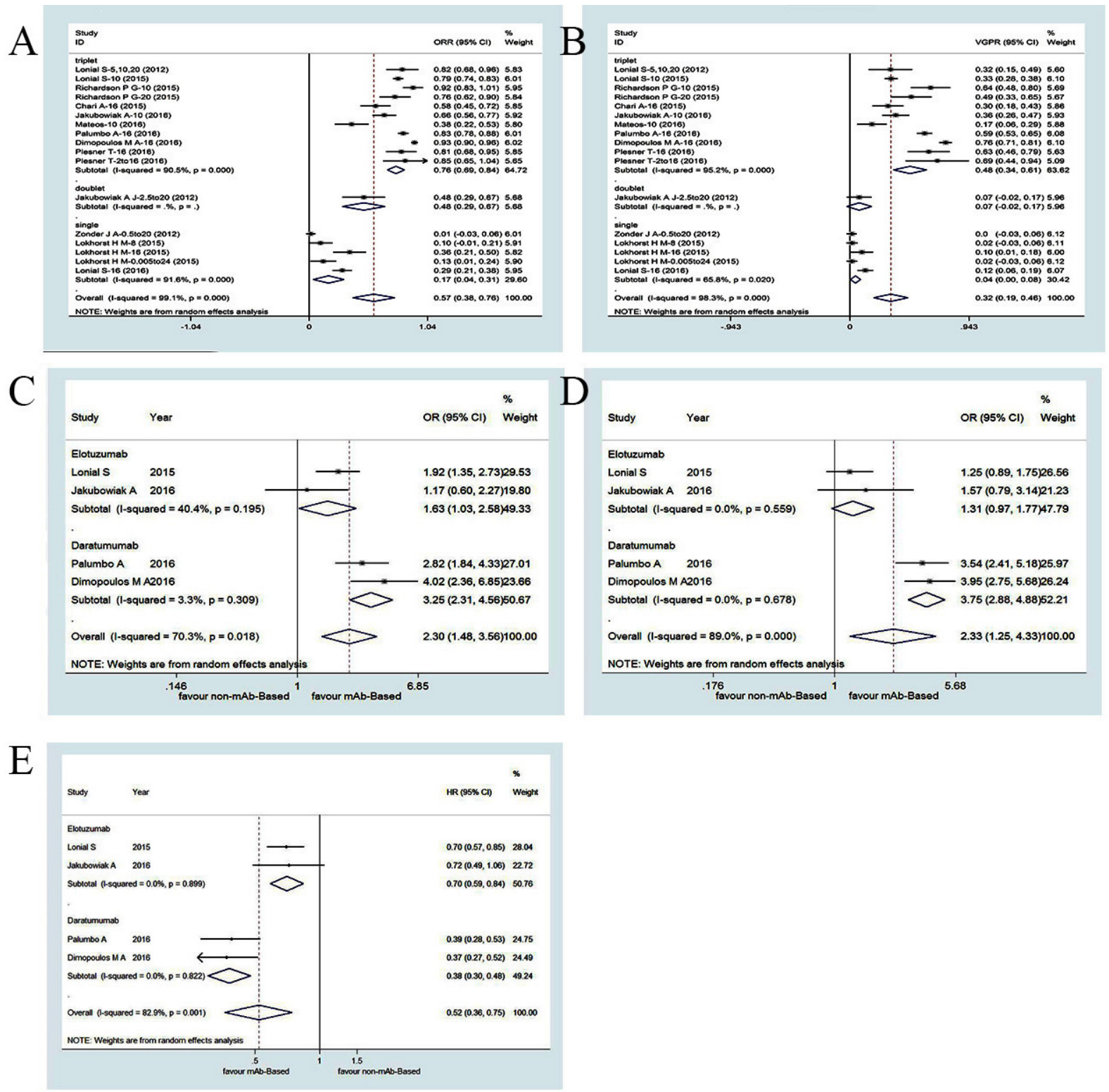

Figure 2: Meta-analysis of the efficacy of mAbs-based regimens in patients with RRMM: (A) overall response rate of mAbs-based single, doublet and triplet regimens;(B) at least very good partial response of mAb-based single, doublet and triplet regimens;(C) odds ratio of overall response of $\mathrm{mAb}$-based triplet compared with controlled arm; (D) odds ratio of at least very good partial response of $\mathbf{m A b s}$-based triplet compared with controlled arm;(E) hazard ratios for progression free survival of mAbs-based triplet compared with controlled arm. ORR, overall response rate; VGPR, very good partial response; OR, odds ratio; HR, hazard ratio; CI, confidence interval 
Table 4A: Pooled analysis of common grade at least 3 adverse events

\begin{tabular}{|c|c|c|c|c|}
\hline Adverse event & No. of trials & $\begin{array}{l}\text { Rate ratio in E. } \\
(\%, 95 \% \text { CI })\end{array}$ & $\begin{array}{l}\text { Rate ratio in D. } \\
(\%, 95 \% \mathrm{CI})\end{array}$ & $\begin{array}{l}\text { Rate ratio in mAbs } \\
(\%, 95 \% \text { CI })\end{array}$ \\
\hline \multicolumn{5}{|l|}{ Hematologic } \\
\hline Neutropenia & $10[5,11-13,27,28,30,33-35]$ & $25(13,38)$ & $34(15,53)$ & $30(17,43)$ \\
\hline Lymphopenia & $6[5,11,13,27,30,33]$ & $41(-2,84)$ & $7(4,11)$ & $24(0,49)$ \\
\hline Thrombocytopenia & $11[5,11-13,27,28,30,32-35]$ & $16(11,20)$ & $18(6,29)$ & $17(10,23)$ \\
\hline Anemia & $11[5,11-13,27-30,32-35]$ & $11(5,18)$ & $14(8,20)$ & $13(9,17)$ \\
\hline Leukopenia & $4[27,30,33,35]$ & $9(3,14)$ & $9(-4,21)$ & $8(2,14)$ \\
\hline \multicolumn{5}{|l|}{ Nonhematologic } \\
\hline Pneumonia & $5[5,13,28,30,35]$ & $9(1,16)$ & $8(6,10)$ & $8(6,10)$ \\
\hline Fatigue & $11[5,11-13,27,28,30,32-35]$ & $7(5,10)$ & $4(2,6)$ & $5(3,7)$ \\
\hline Peripheral neuropathy & $5[5,27,30-32]$ & $7(2,11)$ & $5(2,7)$ & $5(3,7)$ \\
\hline Diarrhoa & $10[5,11,13,27,28,30,32-35]$ & $6(4,8)$ & $3(2,5)$ & $4(3,6)$ \\
\hline Pyrexia & $11[5,11,13,27,28,30-35]$ & $3(1,4)$ & $1(1,2)$ & $2(1,2)$ \\
\hline Back pain & $7[11,13,27,28,31,33,34]$ & $3(-1,6)$ & $2(1,3)$ & $2(0,3)$ \\
\hline
\end{tabular}

Table 4B: Meta-analysis of mAb-based therapies common grade at least 3 adverse events

\begin{tabular}{|l|c|c|c|c|c|}
\hline \multicolumn{1}{|c|}{ Adverse event } & No. of trials & $\begin{array}{c}\text { Events in } \\
\text { mAb arm }\end{array}$ & Events in control arm & Risk ratio(95\% CI) & $\boldsymbol{p}$ \\
\hline Hematologic & & & & & \\
\hline Neutropenia & $3[5,11,13]$ & $285 / 844$ & $252 / 835$ & $1.36(0.77,2.41)$ & 0.293 \\
\hline Lymphopenia & $3[5,11,13]$ & $282 / 844$ & $170 / 835$ & $1.83(1.16,2.87)$ & $0.009 *$ \\
\hline Thrombocytopenia & $4[5,11,13,32]$ & $214 / 919$ & $193 / 910$ & $1.02(0.75,1.39)$ & 0.886 \\
\hline Anemia & $4[5,11,13,32]$ & $135 / 919$ & $165 / 910$ & $0.81(0.66,1)$ & 0.051 \\
\hline Nonhematologic & & & & & $0.90(0.6,1.34)$ \\
\hline Pneumonia & $2[5,13]$ & $42 / 526$ & $46 / 518$ & $1.42(0.89,2.26)$ & 0.137 \\
\hline Fatigue & $4[5,11,13,32]$ & $59 / 919$ & $42 / 910$ & $0.71(0.4,1.27)$ & 0.251 \\
\hline Peripheral Neuropathy & $2[5,32]$ & $18 / 318$ & $25 / 312$ & $1.61(1.01,2.56)$ & $0.046 *$ \\
\hline Diarrhoa & $4[5,11,13,32]$ & $46 / 919$ & $28 / 910$ & $0.90(0.46,1.75)$ & 0.749 \\
\hline Pyrexia & $4[5,11,13,32]$ & $16 / 919$ & $19 / 910$ & $0.63(0.27,1.50)$ & 0.298 \\
\hline Insomnia & $4[5,11,13,32]$ & $8 / 919$ & $14 / 910$ & & 0.600 \\
\hline
\end{tabular}

Table 4C: Meta-analysis of daratumumab-based therapies common grade at least 3 adverse events

\begin{tabular}{|l|c|c|c|c|c|}
\hline \multicolumn{1}{|c|}{ Adverse event } & No. of trials & Events in D. arm & Events in control arm & Risk ratio(95\% CI) & $\boldsymbol{p}$ \\
\hline Hematologic & & & & & \\
\hline Neutropenia & $2[5,13]$ & $178 / 526$ & $114 / 518$ & $1.918(0.90,4.09)$ & 0.092 \\
\hline Lymphopenia & $2[5,13]$ & $38 / 526$ & $16 / 518$ & $2.31(0.93,5.70)$ & 0.07 \\
\hline Thrombocytopenia & $2[5,13]$ & $146 / 526$ & $116 / 518$ & $1.82(0.82,1.71)$ & 0.358 \\
\hline Anemia & $2[5,13]$ & $70 / 526$ & $93 / 518$ & $0.746(0.53,1.05)$ & 0.095 \\
\hline Nonhematologic & & & & & $0.898(0.60,1.34)$ \\
\hline Pneumonia & $2[5,13]$ & $42 / 526$ & $46 / 518$ & 0.600 \\
\hline Fatigue & $2[5,13]$ & $29 / 526$ & $15 / 518$ & $1.87(0.997,3.52)$ & 0.051 \\
\hline Peripheral neuropathy & $1[5]$ & $11 / 243$ & $16 / 237$ & $0.671(0.32,1.42)$ & 0.294 \\
\hline Diarrhoa & $2[5,13]$ & $24 / 526$ & $12 / 518$ & $1.943(0.98,3.86)$ & 0.058 \\
\hline
\end{tabular}


Table 4D: Meta-analysis of elotuzumab-based therapies common grade at least 3 adverse events

\begin{tabular}{|l|c|c|c|c|c|}
\hline \multicolumn{1}{|c|}{ Adverse event } & No. of trials & Events in E. arm & Events in control arm & Risk ratio(95\% CI) & $p$ \\
\hline Hematologic & & & & & \\
\hline Neutropenia & $1[11]$ & $107 / 318$ & $138 / 317$ & $0.773(0.63,0.94)$ & $0.011^{*}$ \\
\hline Lymphopenia & {$[11]$} & $244 / 318$ & $154 / 317$ & $1.579(1.39,1.80)$ & $0.00^{*}$ \\
\hline Thrombocytopenia & $2[11,32]$ & $68 / 393$ & $77 / 392$ & $0.829(0.51,1.34)$ & 0.439 \\
\hline Anemia & $2[11,32]$ & $65 / 393$ & $72 / 392$ & $0.899(0.66,1.22)$ & 0.49 \\
\hline Non-hematologic & & & & & \\
\hline Fatigue & $2[11,32]$ & $30 / 393$ & $27 / 392$ & $1.092(0.66,1.81)$ & 0.731 \\
\hline Peripheral neuropathy & $1[32]$ & $7 / 75$ & $9 / 75$ & $0.778(0.31,1.98)$ & 0.598 \\
\hline Diarrhoa & $2[11,32]$ & $22 / 393$ & $16 / 392$ & $1.366(0.13,2.57)$ & 0.333 \\
\hline Insomnia & $2[11,32]$ & $7 / 393$ & $9 / 392$ & $0.776(0.30,2.06)$ & 0.611 \\
\hline Pyrexia & $2[11,32]$ & $8 / 393$ & $12 / 392$ & $0.609(0.14,2.65)$ & 0.508 \\
\hline
\end{tabular}

mAb: monoclonal antibody; D.:daratumumab; E.:elotuzumab; *: significantly difference between groups in adverse events $(P$ $<0.05)$.

was $29 \%$ (95\% CI: $15-44 \%)$ in a pooled analysis (Figure 3A-B). In a subgroup analysis (triplet/doublet/single), the ORR was $73 \%(95 \%$ CI: $61-84 \%)$ for triplet regimens, 48\% (95\% CI: 29-67\%) for doublet regimens, and $0 \%$ (95\% CI: $-3-6 \%)$ for single regimens, which failed to elicit a significant response in RRMM patients. These results indicated that triplet regimens were superior to doublet and single regimens. The same trend was observed in the at least VGPR. The at least VGPR was 38\% (95\% CI: $27-48 \%)$ for triplet regimens, 7\% (95\% CI: -2-17\%) for doublet regimens, and $0 \%(95 \% \mathrm{CI}:-3-6 \%)$ for single regimens, also suggesting that triplet regimens were the most efficacious.

We analyzed the ORs and HRs reported in two head-to-head RTCs (798 RRMM patients) that compared elotuzumab-based triplet regimens versus nonelotuzumab-based regimens (Figure 2C-E) [11, 32]. The overall weighted ORs for the ORR and at least VGPR were 1.63 (95\% CI: 1.03-2.58) and 1.31 (95\% CI: 0.97-1.77), respectively. The overall weighted PFS HR was 0.70 (95\% CI: 0.59-0.84). The efficacy of elotuzumab triplet regimens did not significantly differ from that of nonelotuzumab-based regimens. Elotuzumab triplet regimens had better efficacy profiles (ORR and at least VGPR) compared to doublet and single regimens. Single regimens had little efficacy in RRMM patients. These meta-analysis results indicated elotuzumab triplet regimens were more effective than non-mAb-based regimens based on the ORR, at least VGPR, and PFS.

\section{Efficacy of daratumumab-based regimens}

There were six studies (869 patients) that evaluated the efficacy of daratumumab for the treatment of RRMM based on the ORR and at least VGPR [5, 12, 13, 33-35].
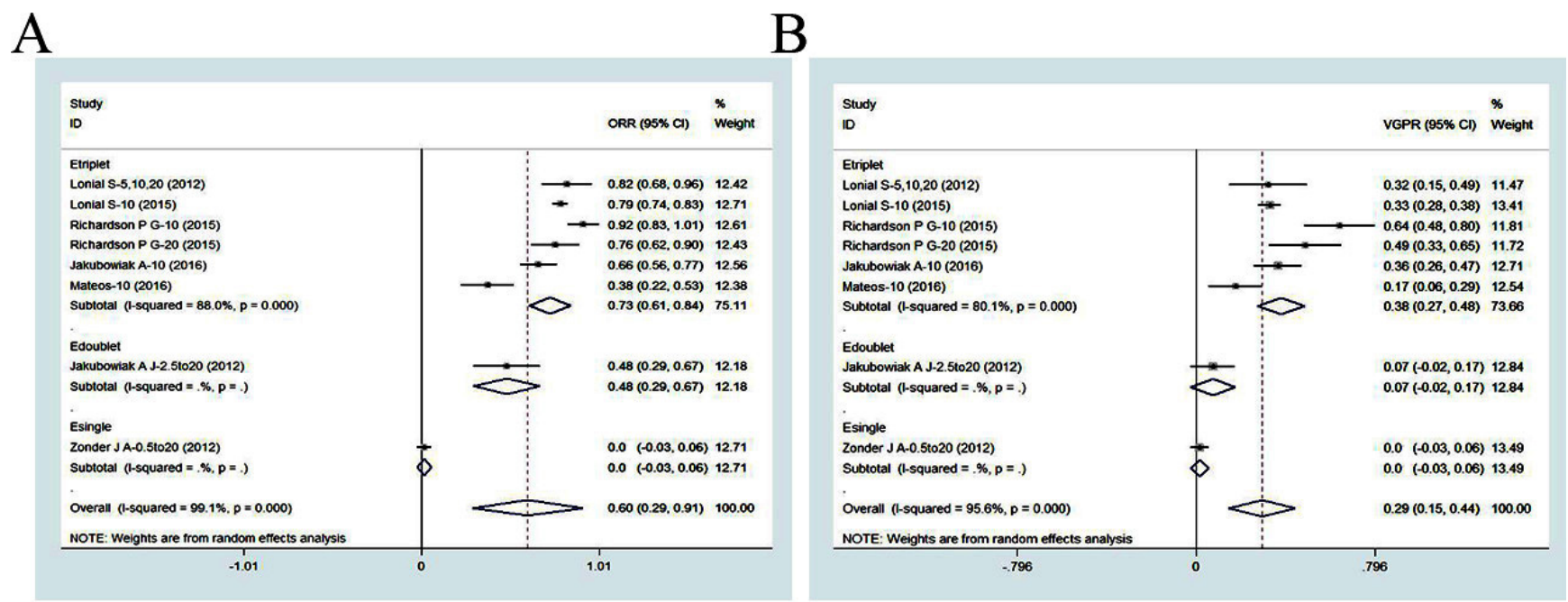

Figure 3: Meta-analysis of the efficacy of elotuzumab-based regimens in patients with RRMM: (A) overall response rate of elotuzuamb-based single, doublet and triplet regimens; (B) at least very good partial response of elotuzumabbased single, doublet and triplet regimens. ORR, overall response rate; VGPR, very good partial response; CI, confidence interval. Etriplet, elotuzumab-based triplet regimen; Edoublet, elotuzumab-based doublet regimen; Esingle, elotuzumab-based single regimen 
The ORR was 54\% (95\% CI: 33-76\%) and the at least VGPR was 35\% (95\% CI: $13-57 \%$ ) in a pooled analysis (Figure 4A-B). In a subgroup analysis (triplet/single), the ORR was $81 \%$ (95\% CI: 71-91\%) for triplet regimens compared to $22 \%$ (95\% CI: $10-33 \%$ ) for single regimens, indicating the triplet regimens were more effective. The same trend was observed in the at least VGPR. The at least VGPR was 59\% (95\% CI: 44-75\%) for triplet regimens and $6 \%(95 \% \mathrm{CI}: 0-11 \%)$ for single regimens, indicating triplet regimens were more favorable. Since $16 \mathrm{mg} / \mathrm{kg}$ is the approved dosage of daratumumab [11, 12], two clinical trials were excluded from our analysis (daratumumab single regimens with non-16 $\mathrm{mg} / \mathrm{kg}$ doses). A pooled analysis after exclusion showed that the ORR and at least VGPR of a daratumumab-based single regimen (16 $\mathrm{mg} / \mathrm{kg}$ ) were $31 \%$ (95\% CI: $24-38 \%)$ and $11 \%(95 \%$ CI: 6-16\%), respectively. Notably, all enrolled patients in this daratumumab-based single regimen subgroup analysis were heavily pretreated MM patients with highly refractory disease. Interestingly, elotuzumab had no effect in heavily pretreated MM patients, which highlighted the effectiveness of daratumumab (Figure 4C-D) [29].

We next analyzed the ORs and HRs reported in two head-to-head RCTs (1,067 patients) that evaluated daratumumab triplet regimens (Figure 2C-E) [5, 13]. The overall weighted ORs of the ORR and at least VGPR were 3.25 (95\% CI: 2.31-4.56) and 3.75 (95\% CI: 2.88-4.88), respectively, while the PFS HR was 0.38 (95\% CI: $0.30-0.48$ ) for daratumumab-based triplet regimens compared to non-daratumumab-based regimens. These results indicated that daratumumab-based triplet regimens had favorable effects on ORR and VGPR compared to single regimens, although single regimens at the optimal dosage were also effective. Daratumumab-based triplet regimens were more effective than non-daratumumabbased regimens according to the ORR, at least VGPR, and PFS. Daratumumab monotherapy $(16 \mathrm{mg} / \mathrm{kg})$ has a remarkable efficacy profile for heavily pretreated MM patients. A summary of patient responses and survival outcomes is shown in Table 3.

\section{Safety}

We performed a pooled analysis to analyze the rate ratio of grade $3 / 4$ adverse events in all included trials.
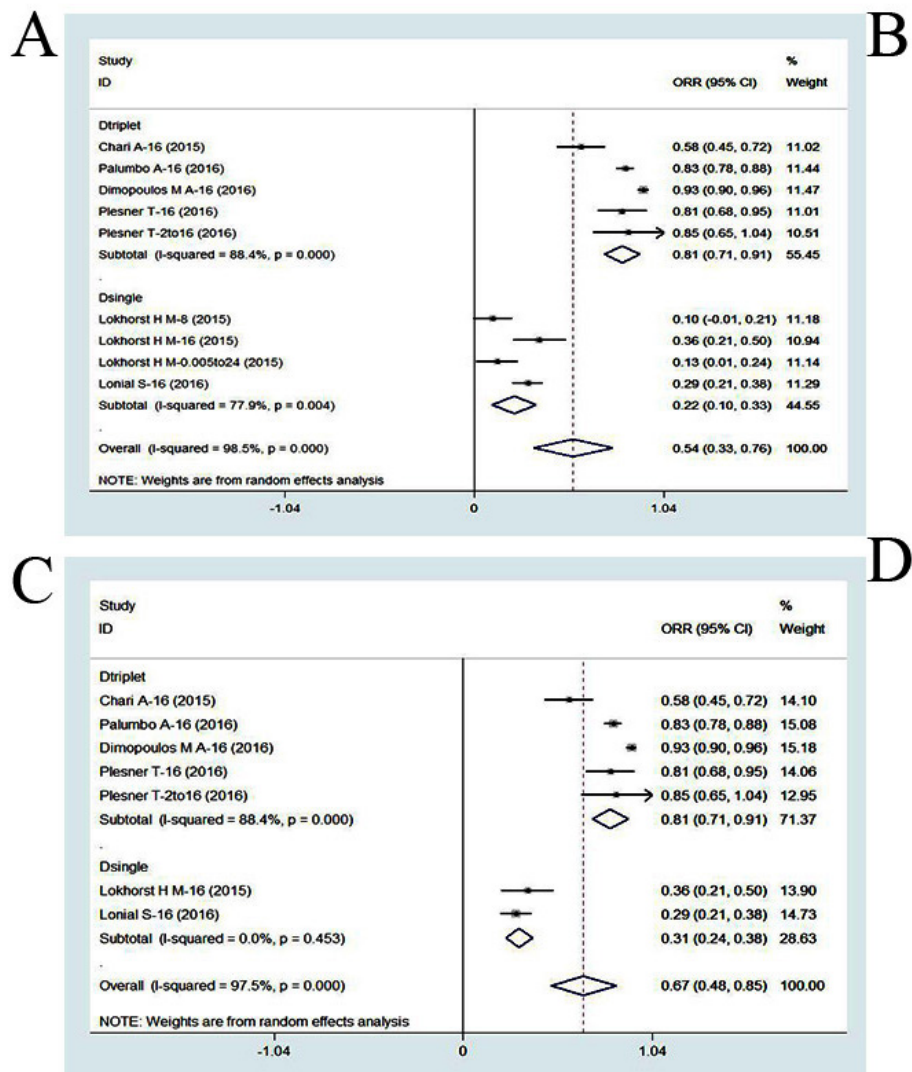

B

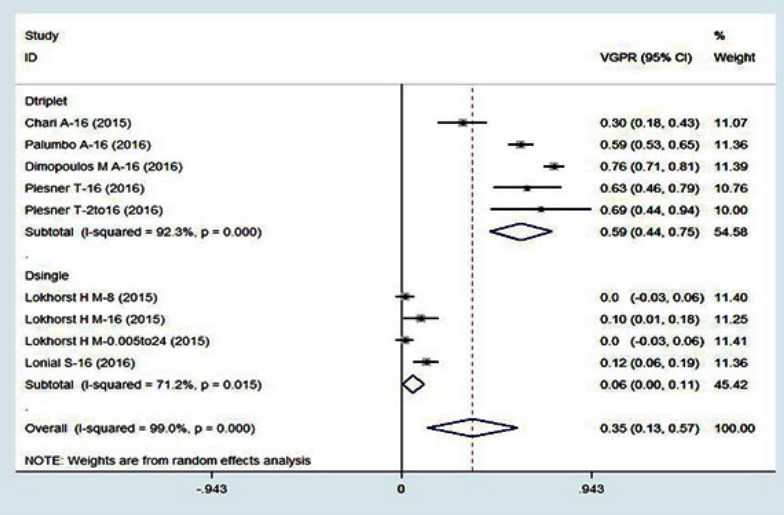

D

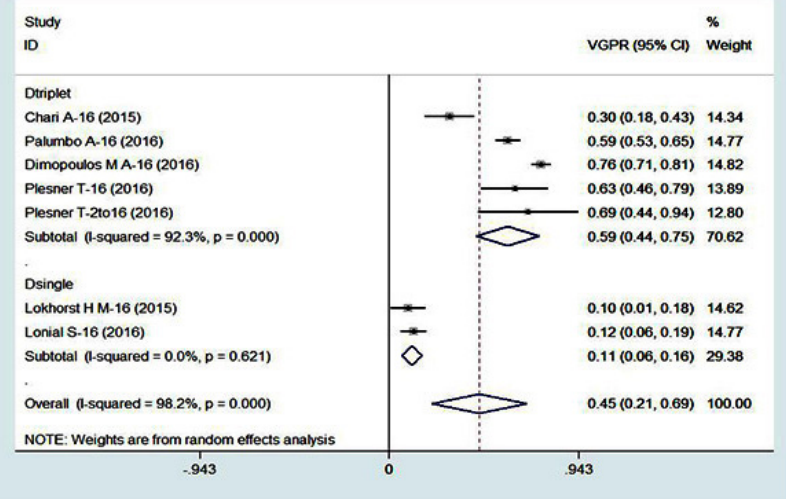

Figure 4: Meta-analysis of the efficacy of daratumumab-based regimens in patients with RRMM:(A) overall response rate of daratumumab-based single and triplet regimens;(B) at least very good partial response of daratumumab-based single and triplet regimens;(C) overall response rate of daratumumab-based monotherapy (16mg/kg);(D) at least very good partial response of daratumumab-based monotherapy $(\mathbf{1 6 m g} / \mathbf{k g})$. ORR, overall response rate; VGPR, very good partial response;CI, confidence interval.Dtriplet, daratumumab-based triplet regimen; Dsingle, daratumumab-based single regimen. 
The most frequent hematological adverse events were neutropenia (30\%, 95\% CI: $17-43 \%)$, lymphopenia (24\%, 95\% CI: 0-49\%), and thrombocytopenia (17\%, 95\% CI: 10-23\%). The most common non-hematological adverse events included pneumonia (8\%, 95\% CI: 6-10\%) and fatigue $(5 \%, 95 \% \mathrm{CI}: 3-7 \%)$ (Table $4 \mathrm{~A})$. We also performed a meta-analysis to analyze the risk ratios of grade 3/4 adverse events in four RCTs that compared mAband non-mAb-based regimens (Table 4B). No differences in most grade $3 / 4$ adverse events were detected with the exception of lymphopenia (risk ratio: $1.83,95 \% \mathrm{CI}: 1.16$ $2.87, \mathrm{P}=0.009$ ), and diarrhea (risk ratio: $1.61,95 \% \mathrm{CI}$ : $1.01-2.56, \mathrm{P}=0.046)$. We performed a subgroup analysis (Table 4) to investigate the most common hematological and non-hematological adverse events resulting from daratumumab and elotuzumab treatment. The most frequent hematological adverse events were neutropenia, lymphopenia, and thrombocytopenia. Elotuzmumabbased regimens primarily resulted in lymphopenia and neutropenia, whereas daratumumab-based regimens primarily caused neutropenia and thrombocytopenia. No statistically significant differences were observed with the addition of mAbs to the treatment regimen, with the exception of increased lymphopenia and diarrhea. The same trend was observed after the addition of elotuzumab, with the exception of an increase in lymphopenia. An unexpected decrease in the occurrence of neutropenia was observed with the addition of elotuzumab to the treatment regimens. This may be one advantage of elotuzumab. Additional studies with larger patient populations are necessary to confirm the benefits of elotuzumab. There were no additional grade $3 / 4$ adverse events observed with daratumumab-based regimens.

\section{Infusion-related reactions}

Based on the pooled analysis of the clinical trials included in our study, infusion-related reactions (any grade) were observed in 46\% (95\% CI: $31-60 \%)$ of the patients, and infusion-related reactions (at least grade 3 ) were observed in only $3 \%$ (95\% CI: $2-5 \%)$ of patients. The infusion-related reactions were primarily observed during the first infusion $(92 \%, 95 \% \mathrm{CI}: 87-97 \%)$. The rate of patients who discontinued the trial due to infusionrelated reactions was also very low $(1 \%, 95 \% \mathrm{CI}: 0-1 \%)$.
A

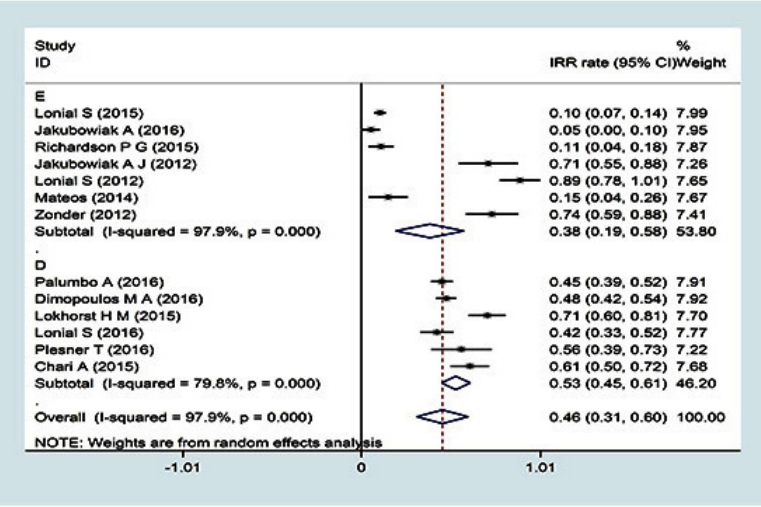

C

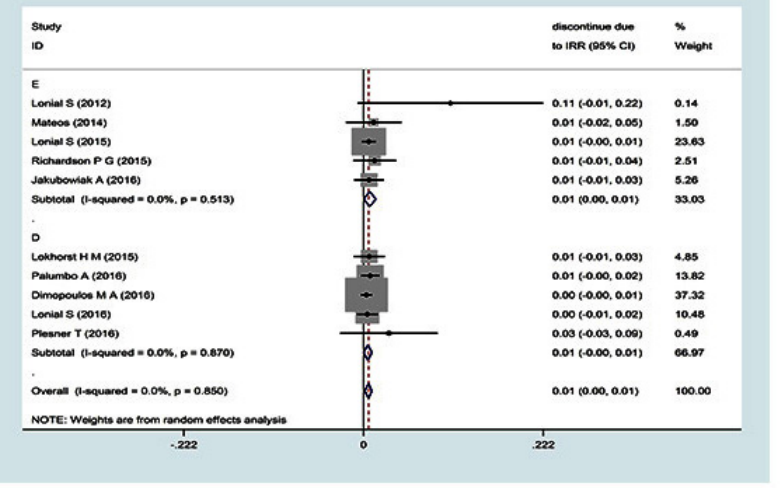

B

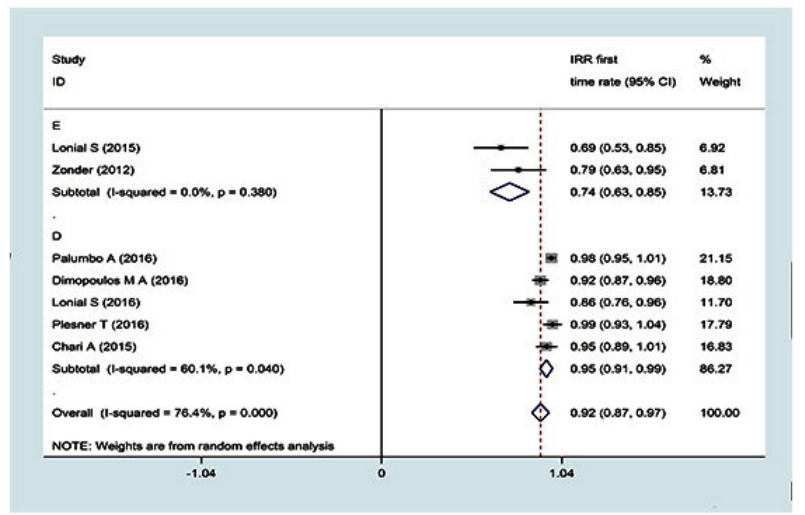

D

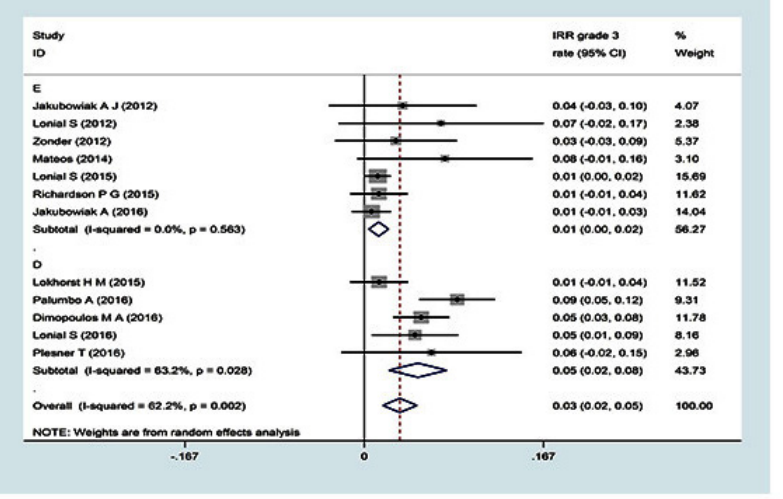

Figure 5: Meta-analysis of the IRRs of mAbs-based regimens in patients with RRMM: (A) any grade infusion-related reactions rate of $\mathrm{mAbs}$;(B) the rate of IRR occurs in first time infusion; (C) grade 3 infusion-related reactions rate of mAbs;(D) the rate of discontinue due to IRRs. IRR, infusion related reactions; CI, confidence interval; E: elotuzumab; D: daratumumab 
In elotuzumab-based clinical trials, infusion-related reactions (any grade) were observed in 38\% (95\% CI: 19 $58 \%$ ) of patients, while infusion-related reactions (at least grade 3 ) were observed in only $1 \%$ (95\% CI: $0-2 \%$ ) of patients. The infusion related reactions primarily occurred during the first infusion $(74 \%, 95 \% \mathrm{CI}$ : $63-85 \%)$. The rate of patients who discontinued due to infusion-related reactions was also very low $(1 \%, 95 \% \mathrm{CI}$ : $0-1 \%)$. In daratumumab-based trials, infusion-related reactions (any grade) were observed in 53\% (95\% CI: 45-61\%) of patients, while infusion-related reactions (at least grade 3 ) were observed in 5\% (95\% CI: $2-8 \%)$ of patients. The infusion-related reactions were primarily observed during the first infusion (95\%, 95\% CI: 91-99\%). The rate of patients who discontinued due to infusion-related reactions was also very low (1\%, 95\% CI: 0-1\%) (Figure $5 \mathrm{~A}-\mathrm{D})$. Collectively, the results indicated that patients treated with $\mathrm{mAb}$-based regimens suffered infusion-related reactions that predominantly occurred during the first infusion, resulting in some patients discontinuing the trials. These reactions were more frequent among patients treated with daratumumab than elotuzumab.
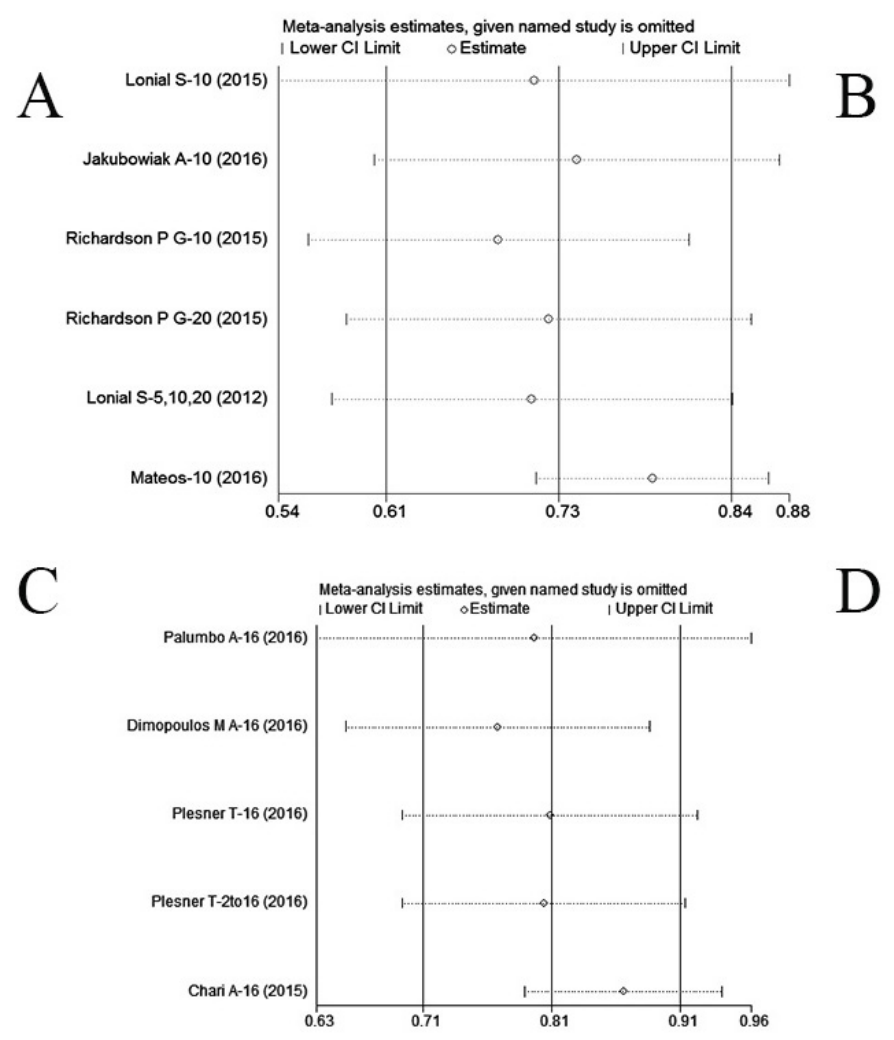

\section{Sensitivity analysis}

We performed a sensitivity analysis of daratumumab and elotuzumab-based triplet regimens using the leaveone-out method in patients with RRMM (Figure 6 A-D). The results indicated that two clinical trials significantly influenced results of the pooled analysis [31, 33] (Figure 6 A-D). These trials involved elotuzumab in combination with thalidomide and dexamethasone [31], and daratumumab in combination with pomalidomide and dexamethasone [33]. This analysis suggested that a mAb in combination with either thalidomide or pomalidomide is not more effective than a $\mathrm{mAb}$ in combination with either lenalidomide or bortezomib.

\section{DISCUSSION}

\section{The efficacy and safety of mAbs for the treatment of RRMM}

In our aggregated analysis, the efficacy and safety trends were reinforced in the pooled population. Our data indicate $\mathrm{mAb}$-based therapy is a superior alternative to

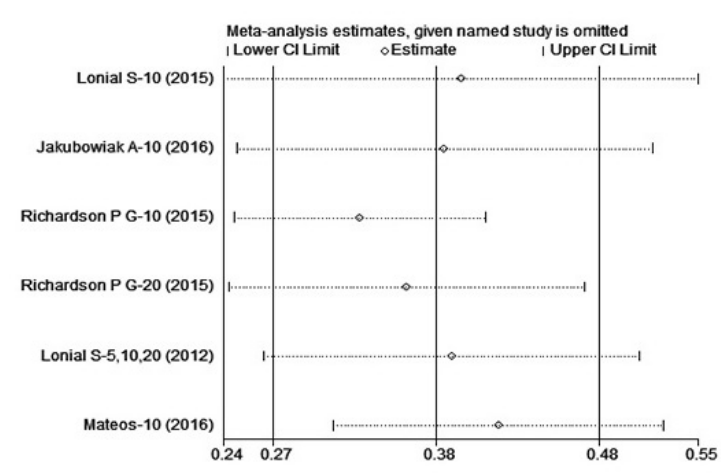

$\mathrm{D}$

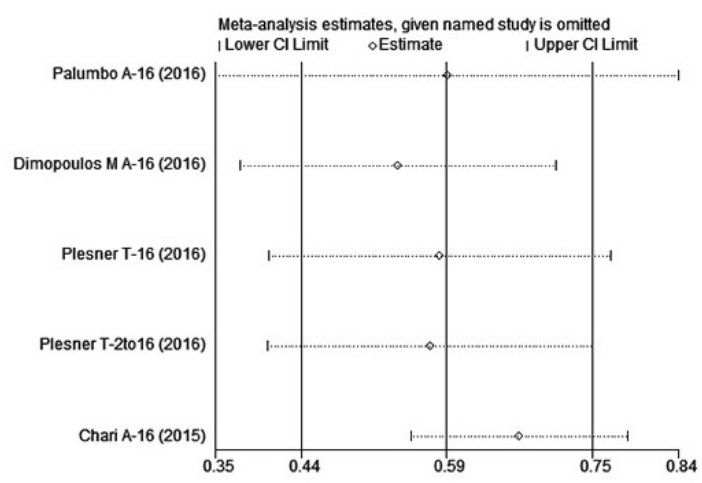

Figure 6: Leave-one-out analysis of the efficacy of daratumumab and elotuzumab-based triplet regimens in patients with RRMM: (A) overall response rate of elotuzumab-based triplet regimens;(B) at least very good partial response of elotuzumab-based triplet regimens;(C) overall response rate of daratumumab-based triplet regimens;(D) at least very good partial response of daratumumab-based triplet regimens. CI, confidence interval. 
Table 5: Summary of response and survival outcomes from novel agent-based regimens

\begin{tabular}{|l|c|c|c|c|c|c|}
\hline \multicolumn{1}{|c|}{ Novel agent } & Regimen & $\begin{array}{c}\text { Median prior } \\
\text { therapy } \\
\text { (range) }\end{array}$ & $\begin{array}{c}\text { ORR } \\
\mathbf{( \% )}\end{array}$ & $\begin{array}{c}\text { At } \\
\text { VGPR } \\
\text { (\%) }\end{array}$ & $\begin{array}{c}\text { least } \\
\text { PFS }\end{array}$ & $\begin{array}{c}\text { Median PFS } \\
\text { (months) }\end{array}$ \\
\hline Triplet & & & & & \\
\hline Daratumumab [13] & $\mathrm{D}+\mathrm{R}+\mathrm{d} / \mathrm{R}+\mathrm{d}$ & $1(1-11) / 1(1-8)$ & $92.9 / 76.4$ & $75.8 / 44.2$ & 0.37 & $\mathrm{NR} / 18.4$ \\
\hline Daratumumab [5] & $\mathrm{D}+\mathrm{V}+\mathrm{d} / \mathrm{V}+\mathrm{d}$ & $2(1-9) / 2(1-10)$ & $82.9 / 63.2$ & $59.2 / 29$ & 0.39 & $\mathrm{NR} / 7.2$ \\
\hline Carfilzomib [8] & $\mathrm{C}+\mathrm{R}+\mathrm{d} / \mathrm{R}+\mathrm{d}$ & $2(1-3)$ & $87.1 / 66.7$ & $69.9 / 40.4$ & 0.69 & $26.3 / 17.6$ \\
\hline Elotuzumab [11] & $\mathrm{E}+\mathrm{R}+\mathrm{d} / \mathrm{R}+\mathrm{d}$ & $2(1-4)$ & $78.5 / 65.5$ & $32.7 / 28$ & 0.70 & $19.4 / 14.9$ \\
\hline Elotuzumab [32] & $\mathrm{E}+\mathrm{V}+\mathrm{d} / \mathrm{V}+\mathrm{d}$ & $1(1-3)$ & $66.2 / 62.6$ & $36.3 / 26.7$ & 0.72 & $9.7 / 6.9$ \\
\hline Ixazomib [10] & $\mathrm{I}+\mathrm{R}+\mathrm{d} / \mathrm{R}+\mathrm{d}$ & $1(1-3)$ & $78.3 / 71.5$ & $48.1 / 39$ & 0.74 & $20.6 / 14.7$ \\
\hline Panobinostat [9] & $\mathrm{P}+\mathrm{V}+\mathrm{d} / \mathrm{V}+\mathrm{d}$ & $1(1-3)$ & $60.7 / 54.6$ & $27.6 / 15.7$ & 0.63 & $12 / 8.1$ \\
\hline Doublet & & & & & & \\
\hline Carfilzomib [39] & $\mathrm{C}+\mathrm{d} / \mathrm{V}+\mathrm{d}$ & $2(1-3)$ & $76.7 / 62.4$ & $54 / 29$ & 0.53 & $18.7 / 9.4$ \\
\hline Elotuzumab [30] & $\mathrm{E}+\mathrm{V}$ & $2(1-3)$ & 48.1 & 7.4 & $\mathrm{NE}$ & 9.46 \\
\hline Pomalidomide [7] & $\mathrm{Po}+\mathrm{d} / \mathrm{d}$ & $5(2-14) / 5(2-17)$ & $31 / 10$ & $4.6 / 0.6$ & 0.48 & $4 / 1.9$ \\
\hline Single & & & & & & \\
\hline Daratumumab-16 & $\mathrm{D}$ & $5(2-14)$ & 31 & 1 & $\mathrm{NE}$ & 2 \\
\hline Carfilzomib [40] & $\mathrm{C}$ & $5(1-20)$ & 28 & 10 & $\mathrm{NE}$ & $\mathrm{NE}$ \\
\hline Pomalidomide [40] & $\mathrm{Po}$ & $5(1-17)$ & 19 & 2 & $\mathrm{NE}$ & $\mathrm{NE}$ \\
\hline Elotuzuamb [29] & $\mathrm{E}$ & $4.5(2-10)$ & 0 & 0 & $\mathrm{NE}$ & $\mathrm{NE}$ \\
\hline
\end{tabular}

E: elotuzumab; D: daratumumab; C: carfilzomib; I: ixazomib; P: panobinostat; Po: pomalidomide; R: lenalidomide; V: bortezomib; d: dexamethasone. Daratumumab-16: daratumumab with optimal dosage of $16 \mathrm{mg} / \mathrm{kg}$; ORR: overall response rate; VGPR: very good partial response; NR: not reach; NE: not estimated.

non-mAb-based therapy because it improves the ORR, at least VGPR, and PFS in RRMM patients, particularly when triplet combination regimens are utilized. Subgroup analysis indicated that $\mathrm{mAb}$-based triplet regimens were superior to doublet regimens, and that doublet regimens were more effective than single regimens. The same trend was also observed for both daratumumab- and elotuzumab-based regimens (Table 3).

Minimal toxicities were associated with the addition of mAbs to the therapeutic regimen. Infusion-related reactions were the most commonly reported adverse events and primarily occurred during the first infusion. We found that they occurred more frequently in daratumumabthan elotuzumab-based clinical trials. Adequate and timely management of infusion-related reactions is important in order to prevent toxicity and treatment discontinuation. If patients experience infusion-related reactions, the infusion should be temporarily interrupted, and the patients should be treated with glucocorticoids or antihistamines at the discretion of the physician [36].

Daratumumab and other CD38-targeting antibodies can interfere with blood typing by binding to CD38 on the surface of red blood cells (RBCs) and leading to a positive indirect Coombs test. Daratumumab interference in pretransfusion tests can be negated by denaturation of surface CD38 on RBCs using the reducing agent dithiothreitol [37]. Patients should undergo extensive RBC antigen phenotyping and screening prior to receiving the first infusion of daratumumab or any other CD38-targeting antibody [38]. Our results demonstrate that novel mAb- based regimens achieve superior responses compared to non-mAb-based regimens, without a risk of toxicity. We performed cross-trial comparisons of newly marketed agents in different combinations in order to provide more practical suggestions for clinical decision-making.

\section{The best option among novel agent-based triplet regimens for $R R M M$}

Despite confounding caused by different study designs and populations, we conducted a cross-trial comparative analysis to determine the best option among the novel agent-based triplet regimens for RRMM treatment. We ranked the regimens based on the efficacy profiles in the RCTs that investigated the combination of a novel agent and traditional doublet therapy (Table 5). A total of five novel agent-based triplet regimens were evaluated. The daratumumab-based triplet regimen was expected to be the most potent of the regimens.

We found that lenalidomide-based regimens were superior to bortezomib, thalidomide, or pomalidomidebased triplet regimens since the efficacy of daratumumab in combination with lenalidomide plus dexamethasone was superior to daratumumab in combination with dexamethasone plus either pomalidomide or bortezomib. Similarly, the efficacy of elotuzumab in combination with lenalidomide plus dexamethasone was superior to elotuzumab in combination with dexamethasone plus either bortezomib or thalidomide (Table 5 and Figure 6). 
Table 6: Monoclonal antibodies being evaluated in multiple myeloma

\begin{tabular}{|l|c|c|}
\hline \multicolumn{1}{|c|}{ Antibody } & Target & Phase \\
\hline Isatuximab (SAR650984) & CD38 & III \\
\hline MOR202 & CD38 & I/II \\
\hline Milatuzumab & CD74 & I/II \\
\hline Indatuximab ravtansine (drug conjugate) & CD138 & II \\
\hline Tabalumab & B-cell activating factor & II \\
\hline Siltuximab & IL6 & I \\
\hline Lucatumumab & CD40 & I \\
\hline Dacetumumab & CD40 & II \\
\hline BHQ880 & DKK1 & IIa \\
\hline Sotatercept (RAP-011) & Activin receptor ligand trap & I \\
\hline huN901-DM1 (drug conjugate) & CD56 & II/III \\
\hline Pembrolizumab & PD1 & II/III \\
\hline Nivolumab & PD1 & I \\
\hline Atezolizumab & CD274 (PD-L1) & \\
\hline
\end{tabular}

IL6, interleukin 6; PD1, programmed cell death 1; PD-L1, programmed cell death ligand 1.

Thus, daratumumab in combination with lenalidomide plus dexamethasone is currently the best option among all triplet regimens for RRMM.

\section{The best option among novel agent-based doublet regimens for RRMM}

A similar cross-trial comparison was performed to identify the best option among the novel agent-based doublet regimens. We evaluated three different novel agent-based doublet regimens. The regimens were ranked based on the efficacy profiles (Table 5). Daratumumab was not evaluated because there was a lack of data for doublet regimens. Among the doublet agent-based regimens, traditional agents such as bortezomib or lenalidomide plus dexamethasone showed reasonable efficacy. Additional head-to-head clinical trials are required to compare the efficacy between traditional agent-based doublet regimens to novel agent-based doublet regimens. Currently, the best option among novel agent-based doublet regimens is carfilzomib plus dexamethasone.

\section{The best option among novel agent-based single regimens for $R R M M$}

The meta-analysis results demonstrated that daratumumab monotherapy ( $16 \mathrm{mg} / \mathrm{kg}$ dose) yielded an ORR of $31.1 \%$ and at least VGPR of $11.5 \%$. It was therefore ranked first among novel single agents. Since all of the trials analyzed included heavily treated MM patients, daratumumab at a dose of $16 \mathrm{mg} / \mathrm{kg}$ was superior to the other novel single agents among these patients. In contrast, elotuzuamb monotherapy had no effect in these patients. A summary of the responses and survival outcomes of patients treated with the novel agent-based regimens is shown in Table 5 .

\section{Limitations and outlook}

Our meta-analysis had several limitations including high study heterogeneity. Although all clinical trials were talked about the response and adverse events of elotuzumab or daratumumab, the inclusion criteria of the clinical trials included in the meta-analysis significantly differed. Second, long-term observation and follow-up is needed in order to analyze overall survival (OS) and confirm the efficacy and safety of the various regimens. Thirdly, language bias may occur because of language barriers as we only searched among English publications. Finally, the results of the cross-trial comparison may be confounded by differences in study design and context. Additional data from head-to-head comparisons between the various triplet regimens is required to inform clinical decision-making [41]. RCTs and follow-up studies are ongoing and are necessary to validate our findings.

mAb-based therapy is a critical strategy for cancer treatment. It is aimed at engaging or augmenting the immune system to target cancer cells [42]. Our results indicate that triplet regimens that include a $\mathrm{mAb}$, particularly daratumumab, are more effective than doublet and single regimens in RRMM. However, triplet regimens are associated with higher costs. Therefore, further analysis of the cost-effectiveness of triplet and doublet regimens is highly recommended. We demonstrated that both novel mAbs had reasonable efficacy and safety profiles. Daratumumab was highly effective in both triplet and single regimens in both general and heavily pretreated MM patients. However, possible infusionrelated reactions, interference with blood tests, and drug resistance should be considered when treating patients with daratumumab.

The effects of daratumumab suggests that CD38 may be a prominent therapeutic target. Interestingly, two new agents, MOR202 (MOR03087) and isatuximab 
(SAR650984), which target CD38, have already demonstrated favorable efficacy and safety in the clinic. MOR202 has been evaluated as monotherapy and in combination with either pomalidomide or lenalidomide in ongoing phase I/IIa clinical trials (NCT01421186). A positive ORR and VGPR were observed with MOR202 monotherapy in approximately $31 \%$ and $13 \%$ of patients, respectively. The duration of MOR202 infusion is 2 hours, which is relatively brief in comparison to daratumumab and the rate of infusion-related reactions is low $(10 \%$ compared to $48 \%$ with daratumumab [43]. Isatuximab demonstrated significant activity as a single agent in a phase I trial involving 35 patients with RRMM who had received a median of six prior lines of therapy. At doses above $10 \mathrm{mg} / \mathrm{kg}$, the ORR and VGPR were $33 \%$ and $11 \%$, respectively [44]. It also demonstrated clear activity as a single agent in a phase II trial involving 97 patients with RRMM who had received a median of five prior lines of therapy resulting in an ORR of $24 \%$. Infusion-related reactions occurred in $49 \%$ of the patients [45]. Since both mAbs have promising efficacy and safety profiles, they may be particularly useful for RRMM treatment, especially in heavily pretreated patients.

Daratumumab exerts its anti-cancer effects through promoting cellular cytotoxicity [16, 17], phagocytosis [17, 18], and induction of apoptosis [17, 19]. Besides, according to the recent study, it also depletes immunosuppressive regulatory $\mathrm{T}$ cells, $\mathrm{B}$ cells, and myeloid-derived suppressor cells that are CD38-positive [20]. Cytotoxic T-cell number, activation and clonal expansion increased after daratumumab treatment in heavily pretreated MM patients [20]. Thus, the mechanism of anti-CD38 novel agent may suggest that they possess an outstanding effect on tumor cells than other traditional regimens in triplet regimens for RRMM and single regimen for heavily pretreated MM patients.

The unique mechanism of action of daratumumab and the minimal toxicity of this agent suggest that it may be the most effective of these agents for RRMM treatment. The treatment against MM is continuously evolving due to the development of new mAbs, especially CD38 and PD1 [46, 47] (Table 6). Future clinical studies of these new agents will reveal which combinations are the most effective for maintenance therapy, newly diagnosed multiple myeloma, and RRMM [46].

\section{MATERIALS AND METHODS}

\section{Search strategy}

This study was performed in accordance with the preferred reporting items for systematic reviews and metaanalyses (PRISMA) statement [48, 49]. We queried the EMBASE, MEDLINE, Cochrane Library, Clinicaltrials. gov, American Society of Hematology, American Society of Clinical Oncology, and European Hematology Association databases to identify clinical trials that investigated the outcomes of patients who received elotuzumab- and daratumumab-based therapy for RRMM. The following medical terms were used in the search: (1) daratumumab, (2) darzalex, (3) elotuzumab, (4) empliciti, (5) 1 OR 2 OR 3 OR 4, (6) relapsed, (7) refractory, (8) 6 OR 7, (9) myeloma, and (10) 5 AND 8 AND 9 in the study titles or abstracts. The search was limited to publications in English. The reference lists of included articles were manually reviewed to identify eligible studies that may have been missed during the initial search. The search results were last updated in November 2016.

\section{Study selection and endpoints}

Studies were eligible for inclusion in the metaanalysis if they met all the following criteria: (1) publication date between January 2005 and November 2016; (2) clinical trial; (3) intervention group containing elotuzumab or daratumumab; (4) investigated patients with RRMM; and (5) at least one of the following data was reported: ORR, VGPR, PFS, or OS. The article with the most recent publication date was selected if multiple publications were available for a given study. All potentially relevant articles were reviewed by two independent investigators (Tiantian Zhang and Sen Wang). Discrepancies in study eligibility were resolved through discussions among investigators. Our primary efficacy endpoints of interest were ORR and at least VGPR, and the other important endpoints were survival (PFS and OS). The safety endpoints were grade 3/4 treatment-related adverse events and infusion-related reactions.

\section{Data extraction}

Study data was collected by two independent reviewers (Tiantian Zhang and Sen Wang), and included the name of the first author, year of publication, trial name, clinical trial phase, median patient age, therapeutic regimen, the median number of prior therapies, number of patients, median follow-up time, median PFS, and OS.

\section{Statistical analysis}

Because there were some phase I and II clinical trials that did not have control arms, we performed a pooled analysis for all clinical trials and a meta-analysis of RCTs $[23,40]$. Rate ratios and 95\% CIs were used to describe clinical outcomes (efficacy and safety) in a single arm according to the ORR and at least VGPR, and adverse events. ORs and $95 \%$ CIs were used to describe clinical outcomes (efficacy) according to the ORR and at least 
VGPR. PFS HRs and 95\% CIs for the intervention versus control arm were representative of treatment efficacy. The RR and associated 95\% CI were used to describe safety based on the effect size.

The meta-analysis was performed using Stata (v14). A random effects model was utilized to calculate the pooled HR, ratio rate, OR, and 95\% CI. Inter-study heterogeneity was estimated using Cochran's Q and $\mathrm{I}^{2}$ tests. A $\mathrm{P}$ value $<0.1$ or an $\mathrm{I}^{2}$ statistic $>50 \%$ was indicative of significant heterogeneity. A random-effects model was selected if the $\mathrm{I}^{2}$ was significant, and a fixedeffects model was selected in all other cases. Clinical trials were classified into subgroups according to the therapeutic regimen: elotuzuamb- or daratumumab-based triplet, doublet, or single-agent. Sensitivity analysis was performed by sequentially excluding individual studies and recalculating the ORR and at least VGPR. Comparisons between three groups were performed with partitions of Pearson's chi-square statistic or Fisher's exact test. Comparisons between two groups were performed using Pearson's chi-square or Fisher's exact tests for categorical variables. Forest and leave-one-out sensitivity analysis plots were generated with Stata (v14). All tests were two-sided and a $\mathrm{P}<0.05$ was considered statistically significant.

\section{Abbreviations}

mAb: Monoclonal antibody; RRMM: relapsed or relapsed and refractory multiple myeloma ; RCT: randomized controlled trial; ORR: overall response rate; VGPR: very good partial response; PI: proteasome inhibitors ; IMiD : immunomodulatory ; NR: not reach; NE: not estimated; HR: hazard ratio; RR: risk ratio; OR: odds ratio; $\mathrm{AE}$ : adverse event; IRR: infusion related reaction; PFS: progression-free survival; OS: overall survival; HDAC- inhibitor: histone deacetylase inhibitor; SLAMF7: signaling lymphocytic activation molecule F7; NA: not available or no target points.

\section{Author contributions}

Study design, literature research, manuscript editing, Tiantian Zhang and Sen Wang; data acquisition: Zhuoru Liang; statistical analysis: Tengfei Lin and Yangqiu Li; revised and polished the manuscript: Jingmei Xie and Lina Zhao; manuscript final version approval: Jie Jiang.

\section{ACKNOWLEDGMENTS}

We are indebted to no one for assistance with the manuscript.

\section{CONFLICTS OF INTEREST}

We are indebted to no conflicts of interest with anyone.

\section{FUNDING}

We did not receive any financial support.

\section{REFERENCES}

1. Zou YD, Sheng ZX, Lu HK, Yu JM. Continuous treatment with new agents for newly diagnosed multiple myeloma. Anti-Cancer Drugs. 2013; 24: 527-33. doi: 10.1097/ CAD.0b013e32836032d5.

2. Siegel RL, Miller KD, Jemal A. Cancer statistics, 2016. CA Cancer J Clin. 2016; 66: 7-30. doi: 10.3322/caac.21332.

3. Rajkumar SV, Harousseau JL, Durie B, Anderson KC, Dimopoulos M, Kyle R, Blade J, Richardson P, Orlowski R, Siegel D, Jagannath S, Facon T, Avet-Loiseau H, et al. Consensus recommendations for the uniform reporting of clinical trials: report of the International Myeloma Workshop Consensus Panel 1. Blood. 2011; 117: 4691-5. doi: 10.1182/blood-2010-10-299487.

4. Anderson $\mathrm{KC}$, Alsina $\mathrm{M}$, Atanackovic D, Biermann JS, Chandler JC, Costello C, Djulbegovic B, Fung HC, Gasparetto C, Godby K, Hofmeister C, Holmberg L, Holstein S, et al. NCCN Guidelines Insights: Multiple Myeloma, Version 3.2016. J Natl Compr Canc Netw. 2016; 14: 389-400.

5. Palumbo A, Chanan-Khan A, Weisel K, Nooka AK, Masszi T, Beksac M, Spicka I, Hungria V, Munder M, Mateos MV, Mark TM, Qi M, Schecter J, et al. Daratumumab, Bortezomib, and Dexamethasone for Multiple Myeloma. $\mathrm{N}$ Engl J Med. 2016; 375: 754-66. doi: 10.1056/ NEJMoa1606038.

6. Usmani S, Ahmadi T, Ng Y, Lam A, Potluri R, Mehra M. Analyses of Real World Data on Overall Survival in Multiple Myeloma Patients with at Least 3 Prior Lines of Therapy Including a PI and an IMiD, or Double Refractory to a PI and an IMiD. Blood. 2015; 126.

7. Miguel JS, Weisel K, Moreau P, Lacy M, Song K, Delforge M, Karlin L, Goldschmidt H, Banos A, Oriol A, Alegre A, Chen C, Cavo M, et al. Pomalidomide plus low-dose dexamethasone versus high-dose dexamethasone alone for patients with relapsed and refractory multiple myeloma (MM-003): a randomised, open-label, phase 3 trial. Lancet Oncology. 2013; 14: 1055-66. doi: 10.1016/S14702045(13)70380-2.

8. Stewart AK, Rajkumar SV, Dimopoulos MA, Masszi T, Spicka I, Oriol A, Hajek R, Rosinol L, Siegel DS, Mihaylov GG, Goranova-Marinova V, Rajnics P, Suvorov A, et al. Carfilzomib, lenalidomide, and dexamethasone for relapsed multiple myeloma. New England Journal of Medicine. 
2015; 372: 142-52. doi: 10.1056/Nejmoa1411321.

9. San-Miguel JF, Hungria VTM, Yoon SS, Beksac M, Dimopoulos MA, Elghandour A, Jedrzejczak WW, Gunther A, Nakorn TN, Siritanaratkul N, Corradini P, Chuncharunee S, Lee JJ, et al. Panobinostat plus bortezomib and dexamethasone versus placebo plus bortezomib and dexamethasone in patients with relapsed or relapsed and refractory multiple myeloma: a multicentre, randomised, double-blind phase 3 trial. Lancet Oncology. 2014; 15: 1195-206. doi: 10.1016/S1470-2045(14)70440-1.

10. Moreau P, Masszi T, Grzasko N, Bahlis NJ, Hansson M, Pour L, Sandhu I, Ganly P, Baker BW, Jackson SR, Stoppa AM, Simpson DR, Gimsing P, et al. Oral Ixazomib, Lenalidomide, and Dexamethasone for Multiple Myeloma. New England Journal of Medicine. 2016; 374: 1621-34. doi: 10.1056/Nejmoa1516282.

11. Lonial S, Dimopoulos M, Palumbo A, White D, Grosicki S, Spicka I, Walter-Croneck A, Moreau P, Mateos MV, Magen H, Belch A, Reece D, Beksac M, et al. Elotuzumab therapy for relapsed or refractory multiple myeloma. N Engl J Med. 2015; 373: 621-31. doi: 10.1056/NEJMoa1505654.

12. Lonial S, Weiss BM, Usmani SZ, Singhal S, Chari A, Bahlis NJ, Belch A, Krishnan A, Vescio RA, Mateos MV, Mazumder A, Orlowski RZ, Sutherland HJ, et al. Daratumumab monotherapy in patients with treatmentrefractory multiple myeloma (SIRIUS): an open-label, randomised, phase 2 trial. Lancet. 2016; 387: 1551-60. doi: 10.1016/S0140-6736(15)01120-4.

13. Dimopoulos MA, Oriol A, Nahi H, San-Miguel J, Bahlis NJ, Usmani SZ, Rabin N, Orlowski RZ, Komarnicki M, Suzuki K, Plesner T, Yoon SS, Ben Yehuda D, et al. Daratumumab, lenalidomide, and dexamethasone for multiple myeloma. N Engl J Med. 2016; 375: 1319-31. doi: 10.1056/NEJMoa1607751.

14. Raje N, Longo DL. Monoclonal antibodies in multiple myeloma come of age. New England Journal of Medicine. 2015; 373: 1264-6. doi: 10.1056/Nejme1509419.

15. Lin P, Owens R, Tricot G, Wilson CS. Flow cytometric immunophenotypic analysis of 306 cases of multiple myeloma. American Journal of Clinical Pathology. 2004; 121: 482-8. doi: 10.1039/74r4tb90buwh27jx.

16. de Weers M, Tai YT, van der Veer MS, Bakker JM, Vink T, Jacobs DC, Oomen LA, Peipp M, Valerius T, Slootstra JW, Mutis T, Bleeker WK, Anderson KC, et al. Daratumumab, a novel therapeutic human CD38 monoclonal antibody, induces killing of multiple myeloma and other hematological tumors. J Immunol. 2011; 186: 1840-8. doi: 10.4049/jimmunol.1003032.

17. van Bueren JL, Jakobs D, Kaldenhoven N, Roza M, Hiddingh S, Meesters J, Voorhorst M, Gresnigt E, Wiegman L, Buijsse AO, Andringa G, Overdijk MB, Doshi P, et al. Direct in vitro comparison of daratumumab with surrogate analogs of CD38 antibodies MOR03087, SAR650984 and Ab79. Blood. 2014; 124.

18. Overdijk MB, Verploegen S, Bogels M, van Egmond M, van Bueren JJL, Mutis T, Groen RWJ, Breij E, Martens ACM, Bleeker WK, Parren PW. Antibody-mediated phagocytosis contributes to the anti-tumor activity of the therapeutic antibody daratumumab in lymphoma and multiple myeloma. Mabs. 2015; 7: 311-20. doi: 10.1080/19420862.2015.1007813.

19. Jansen JHM, Boross P, Overdijk MB, van Bueren JJL, Parren PW, Leusen JHW. Daratumumab, a human CD38 antibody induces apoptosis of myeloma tumor cells via fc receptor-mediated crosslinking. Blood. 2012; 120.

20. Krejcik J, Casneuf T, Nijhof IS, Verbist B, Bald J, Plesner T, Syed K, Liu K, van de Donk NW, Weiss BM, Ahmadi T, Lokhorst HM, Mutis T, et al. Daratumumab depletes CD38+ immune regulatory cells, promotes T-cell expansion, and skews T-cell repertoire in multiple myeloma. Blood. 2016; 128: 384-94. doi: 10.1182/blood-2015-12-687749.

21. Jelinek T, Hajek R. Monoclonal antibodies - A new era in the treatment of multiple myeloma. Blood Rev. 2016; 30 : 101-10. doi: 10.1016/j.blre.2015.08.004.

22. Collins SM, Bakan CE, Swartzel GD, Hofmeister CC, Efebera YA, Kwon H, Starling GC, Ciarlariello D, Bhaskar S, Briercheck EL, Hughes T, Yu J, Rice A, et al. Elotuzumab directly enhances NK cell cytotoxicity against myeloma via CS1 ligation: evidence for augmented NK cell function complementing ADCC. Cancer Immunol Immunother. 2013; 62: 1841-9. doi: 10.1007/s00262-0131493-8.

23. Liu JD, Sun CY, Tang L, Wu YY, Wang QY, Hu B, Hu Y. Efficacy and safety of panobinostat in relapsed or/and refractory multiple myeloma: meta analyses of clinical trials and systematic review. Scientific Reports. 2016; 6:27361.

24. Parikh K, Purnomo L, Abouzaid S, Chen CC, McGuiness C, Hussein M, Wade R. Pomalidomide or carfilzomib use in patients with relapsed multiple myeloma: real-world treatment patterns, time to next treatment, and economic outcomes. Haematologica. 2016; 101: 603.

25. Qiao SK, Guo XN, Ren JH, Ren HY. Efficacy and safety of lenalidomide in the treatment of multiple myeloma: A systematic review and meta-analysis of randomized controlled trials. Chinese Medical Journal. 2015; 128: 121522. doi: 10.4103/0366-6999.156134.

26. Knopf KB, Duh MS, Lafeuille MH, Gravel J, Lefebvre P, Niculescu L, Ba-Mancini A, Ma E, Shi HL, Comenzo RL. Meta-analysis of the efficacy and safety of bortezomib re-treatment in patients with multiple myeloma. Clinical Lymphoma Myeloma \& Leukemia. 2014; 14: 380-8. doi: 10.1016/j.clml.2014.03.005.

27. Richardson PG, Jagannath S, Moreau P, Jakubowiak AJ, Raab MS, Facon T, Vij R, White D, Reece DE, Benboubker L, Zonder J, Tsao LC, Anderson KC, et al. Elotuzumab in combination with lenalidomide and dexamethasone in patients with relapsed multiple myeloma: final phase 2 results from the randomised, open-label, phase $1 \mathrm{~b}-2$ doseescalation study. Lancet Haematology. 2015; 2: E516-E27. doi: 10.1016/S2352-3026(15)00197-0. 
28. Lonial S, Vij R, Harousseau JL, Facon T, Moreau P, Mazumder A, Kaufman JL, Leleu X, Tsao LC, Westland C, Singhal AK, Jagannath S. Elotuzumab in combination with lenalidomide and low-dose dexamethasone in relapsed or refractory multiple myeloma. Journal of Clinical Oncology. 2012; 30: 1953-9. doi: 10.1200/Jco.2011.37.2649.

29. Zonder JA, Mohrbacher AF, Singhal S, van Rhee F, Bensinger WI, Ding H, Fry J, Afar DE, Singhal AK. A phase 1, multicenter, open-label, dose escalation study of elotuzumab in patients with advanced multiple myeloma. Blood. 2012; 120: 552-9. doi: 10.1182/ blood-2011-06-360552.

30. Jakubowiak AJ, Benson DM, Bensinger W, Siegel DSD, Zimmerman TM, Mohrbacher A, Richardson PG, Afar DEH, Singhal AK, Anderson KC. Phase I trial of anti-CS1 monoclonal antibody elotuzumab in combination with bortezomib in the treatment of relapsed/refractory multiple myeloma. Journal of Clinical Oncology. 2012; 30: 1960-5. doi: 10.1200/Jco.2011.37.7069.

31. Mateos MV, Granell M, Rocafiguera AO, Lopez JM, Blade J, Hernandez MT, Sanchez JM, Gironella M, Lynch M, Bleickardt E, Paliwal P, Singhal A, San Miguel J. A phase Ii single-arm safety study of elotuzumab in combination with thalidomide and low dose dexamethasone in patients with relapsed and/or refractory multiple myeloma. Haematologica. 2014; 99: 361-2.

32. Jakubowiak A, Offidani M, Pegourie B, De La Rubia J, Garderet L, Laribi K, Bosi A, Marasca R, Laubach J, Mohrbacher A, Carella AM, Singhal AK, Tsao LC, et al. Randomized phase 2 study: elotuzumab plus bortezomib/ dexamethasone vs bortezomib/dexamethasone for relapsed/ refractory MM. Blood. 2016; 127: 2833-40. doi: 10.1182/ blood-2016-01-694604.

33. Chari A, Lonial S, Suvannasankha A, Fay JW, Arnulf B, Ifthikharuddin JJ, Qin X, Masterson T, Nottage K, Schecter JM, Ahmadi T, Weiss B, Krishnan A, et al. Open-label, multicenter, phase $1 \mathrm{~b}$ study of daratumumab in combination with pomalidomide and dexamethasone in patients with at least 2 lines of prior therapy and relapsed or relapsed and refractory multiple myeloma. Blood. 2015; 126.

34. Plesner T, Arkenau HT, Gimsing P, Krejcik J, Lemech C, Minnema MC, Lassen U, Laubach JP, Palumbo A, Lisby S, Basse L, Wang J, Sasser AK, et al. Phase 1/2 study of daratumumab, lenalidomide, and dexamethasone for relapsed multiple myeloma. Blood. 2016; 128: 1821-8.

35. Lokhorst HM, Plesner T, Laubach JP, Nahi H, Gimsing P, Hansson M, Minnema MC, Lassen U, Krejcik J, Palumbo A, van de Donk NW, Ahmadi T, Khan I, et al. Targeting CD38 with daratumumab monotherapy in multiple myeloma. N Engl J Med. 2015; 373: 1207-19. doi: 10.1056/ NEJMoa1506348.

36. van de Donk NW, Moreau P, Plesner T, Palumbo A, Gay F, Laubach JP, Malavasi F, Avet-Loiseau H, Mateos MV, Sonneveld P, Lokhorst HM, Richardson PG. Clinical efficacy and management of monoclonal antibodies targeting CD38 and SLAMF7 in multiple myeloma. Blood. 2016; 127: 681-95. doi: 10.1182/blood-2015-10-646810.

37. Chapuy CI, Nicholson RT, Aguad MD, Chapuy B, Laubach JP, Richardson PG, Doshi P, Kaufman RM. Resolving the daratumumab interference with blood compatibility testing. Transfusion. 2015; 55: 1545-54. doi: 10.1111/trf.13069.

38. Oostendorp M, Lammerts van Bueren JJ, Doshi P, Khan I, Ahmadi T, Parren PW, van Solinge WW, De Vooght KM. When blood transfusion medicine becomes complicated due to interference by monoclonal antibody therapy. Transfusion. 2015; 55: 1555-62. doi: 10.1111/trf.13150.

39. Dimopoulos MA, Moreau P, Palumbo A, Joshua D, Pour L, Hajek R, Facon T, Ludwig H, Oriol A, Goldschmidt H, Rosinol L, Straub J, Suvorov A, et al. Carfilzomib and dexamethasone versus bortezomib and dexamethasone for patients with relapsed or refractory multiple myeloma (ENDEAVOR): a randomised, phase 3, open-label, multicentre study. Lancet Oncol. 2016; 17: 27-38. doi: 10.1016/S1470-2045(15)00464-7.

40. Zou Y, Ma X, Yu H, Hu C, Fan L, Ran X. Carfilzomib/ pomalidomide single-agent or in combination with other agents for the management of relapsed/refractory multiple myeloma : a meta-analysis of 37 trials. Oncotarget. $2016 \mathrm{Jul}$ 21. doi: 10.18632/oncotarget.10768. [Epub ahead of print].

41. Rajkumar SV, Kyle RA. Progress in myeloma - a monoclonal breakthrough. N Engl J Med. 2016; 375: 13902. doi: 10.1056/NEJMe1609835.

42. Oliva S, Palumbo A. Monoclonal antibodies for treating multiple myeloma - a new era, new safety considerations? Expert Opin Drug Saf. 2016; 15: 1295-300. doi: 10.1080/14740338.2016.1201061.

43. Marc S. Raab MC. (2016). A phase I/IIa study of the CD38 antibody MOR202 alone and in combination with pomalidomide or lenalidomide in patients with relapsed or refractory multiple myeloma [abstract 1152]. 58th ASH Annual Meeting and exposition,December 3-6,2016 (San Francisco,CA.).

44. Martin TG, Hsu K, Strickland SA, Glenn MJ, Mikhael J, Charpentier E. A phase I trial of SAR650984, a CD38 monoclonal antibody, in relapsed or refractory multiple myeloma. Journal of Clinical Oncology. 2014; 32.

45. Richter J, Martin T, Vij R, Cole C, Atanackovic D, Zonder J, Kaufman J, Mikhael J, Bensinger W, Dimopoulos M, Zimmerman T, Lendvai N, Hari P, et al. Updated data from a phase Ii dose finding trial of single agent isatuximab (Sar650984, Anti-Cd38 Mab) in relapsed/refractory multiple myeloma (Rrmm)[abstract]. Journal of Clinical Oncology. 2016; 34: 2016: 8005.

46. Laubach JP, Paba Prada CE, Richardson PG, Longo DL. Daratumumab, elotuzumab, and the development of therapeutic monoclonal antibodies in multiple myeloma. Clin Pharmacol Ther. 2017; 101: 81-8. doi: 10.1002/ cpt.550.

47. Zagouri F, Terpos E, Kastritis E, Dimopoulos MA. 
Emerging antibodies for the treatment of multiple myeloma. Expert Opin Emerg Drugs. 2016; 21: 225-37. doi: 10.1080/14728214.2016.1186644.

48. Zeng X, Zhang Y, Kwong JS, Zhang C, Li S, Sun F, Niu $\mathrm{Y}, \mathrm{Du} \mathrm{L}$. The methodological quality assessment tools for preclinical and clinical studies, systematic review and meta-analysis, and clinical practice guideline: a systematic review. J Evid Based Med. 2015; 8: 2-10. doi: 10.1111/ jebm. 12141 .

49. Liberati A, Altman DG, Tetzlaff J, Mulrow C, Gotzsche PC, Ioannidis JP, Clarke M, Devereaux PJ, Kleijnen J, Moher D. The PRISMA statement for reporting systematic reviews and meta-analyses of studies that evaluate health care interventions: explanation and elaboration. PLoS Med. 2009; 6: e1000100. doi: 10.1371/journal.pmed.1000100. 